\title{
High-Temperature Alert System using Sensors in Gloves for Hand Safety and Injury Prevention of People with Visual or Touch Sensory Disabilities
}

\author{
${ }^{1}$ Johan A. Grisales, ${ }^{1}$ Fredy E. Hoyos and ${ }^{2}$ John E. Candelo-Becerra \\ ${ }^{1}$ Facultad de Ciencias, Escuela de Fisica, Universidad Nacional de Colombia, Sede Medellin, \\ Calle 59 A No 63-20, 050034 Medellín, Colombia \\ ${ }^{2}$ Departament de Energía Electrica y Automática, Facultad de Minas, \\ Universidad Nacional de Colombia, Sede Medellín, Carrera 80 No 65-223, \\ Campus Robledo, 050041 Medellín, Colombia
}

\begin{abstract}
Providing blind people with tools that allow them to more easily manage different life situations is a necessity. For this reason, this study presents an application of a temperature monitoring system that identifies areas of contact danger by using a glove designed and constructed to alert the wearer of risks of contact. The system consists mainly of contact temperature sensors installed in the glove that communicate with an electronic board. An embedded algorithm is programmed to make temperature risk decisions and to send signals to alert devices. This system can be adapted to other monitoring contexts to emulate and facilitate the senses of touch in people with visual and/or sensory difficulties. Tests were performed by changing the temperature to determine better performance with the system which was achieved by the thermistor.
\end{abstract}

Key words: Injury prevention, blind people, temperature sensor, embedded systems, safety glove, electronic board

\section{INTRODUCTION}

Recent advances in the development of electronic devices have allowed new tools to improve the quality of life. Developers have implemented many tools for blind people (Elmannai and Elleithy, 2017) such as the audio-based multi-touch interaction techniques that enable blind users to access touchscreen applications (Kane et al., 2008). However, users made more errors when using a slide rule input than when using the more familiar button-based system. An interview of 20 participants with visual and motor disabilities and a 1 week study registering the experiences of using mobile devices in their daily lives were performed (Kane et al., 2009). The study revealed how participants used various strategies to adapt mobile devices to perform tasks. Another research introduced, a mobile communication and translation device for deafblind people which translates the hand-touch alphabet, Lorm, a common form of communication used by these people (Gollner et al., 2012). A hardware prototype was created to compose messages via. fabric pressure sensors placed on the palm of the glove to send messages to a handheld device.

By Makino et al. (1996) developed a new guidance system for blind people by using GPS receivers and a mobile phone as an automatic on-line personal guidance function. The test showed that the maximum error of the determined position was $16 \mathrm{~m}$ and the weight of the mobile unit was $<2 \mathrm{~kg}$. By Sjostrom (2001), the researcher used different user interface ideas and games in order to understand the use of haptics in computer interfaces for the blind. Other researchers used mobile and wireless technologies together with text to speech systems and a database to develop an orientation and navigation system using RFID identity tags from cattle slaughtering to create a grid used for navigation (D'Atri et al., 2007).

The literature shows how new devices have been developed to help the visually impaired. However, the need for using technology for people that require assistance has increased and there is no enough devices to help people with other task as preventions for objects that can affect health and this is required specially to obtain economic devices and accessible for most people. Therefore, in this research a glove adapted with temperature sensors is presented as a tool to prevent burn injuries in the blind. The purpose of the tool is to provide a handling aid for people who have visual and/or sensitivity problems. For this, a glove was modified with three $10 \mathrm{k} \Omega$ thermistors located on the front of the fingers which send voltage signals every second to an Arduino board. The signals are converted to temperature values that are displayed on an LCD screen. Three two-color LEDs, located in the front (palm) part of the glove, change from green to red depending on the temperature within the

Corresponding Author: Johan A. Grisales, Facultad de Ciencias, Escuela de Física, Universidad Nacional de Colombia, Sede Medellín, Calle 59 A No 63-20,050034 Medellín, Colombia 
range of $0-80^{\circ} \mathrm{C}$ and emit an audio alarm for high temperatures. Thus, the system is intended to inform the glove user of each temperature that the sensor captures.

\section{MATERIALS AND METHODS}

This study includes the specifications and procedures to design and construct safety gloves for blind people. Initially, the implemented methodology considers the materials necessary for the construction of a glove with sensors. Then, the description of the methodology and the tests conducted with the glove with the two types of temperature sensors are presented.

General procedure: Figure 1 presents the general methodology applied for the glove with a temperature sensor. The system implemented was based on the reading of the temperature in elements that may represent a risk for a blind or sensory-deprived person. The diagram shows that temperature sensors are placed on the glove and signals are transmitted to an embedded system for processing. Then, the system evaluates the temperature risk and provides an alarm (visual or auditory) to warn of the risk. These signals must be interpreted by users who make use of the implemented application as they are warning signs.

Glove design: Figure 2 shows the glove designed to sense the temperature of elements. The primary glove material is a cotton fabric front and back. This style has a common length dimension of $220-270 \mathrm{~mm}$. The glove has been designed to maintain internal wiring for the sensors and LEDs without affecting the user's comfort and functionality. Therefore, three temperature sensors $(10 \mathrm{k} \Omega$ thermistors) were placed in the front (palm) of the glove and three two-color LEDs in the back of the glove where there is the relationship between Sensors S1 and LED L1 located in the little finger, S2 and L 2 located on the thumb and S3 and LED L3 located in the middle finger. This configuration was adopted to allow the user to sense the temperature and observe temperature changes with the brightness of LEDs located in the back of the glove.

Embedded system: Figure 3 shows the implementation of the global circuit using Arduino UNO. Temperature sensors based on $10 \mathrm{k} \Omega$ thermistors are also replaced with integrated circuit temperature sensors (LM335) to evaluate the response of both sensors to different temperatures. In this case, the central Arduino Uno board has the function of interpreting the signals of the analog inputs and sending information to the output elements.

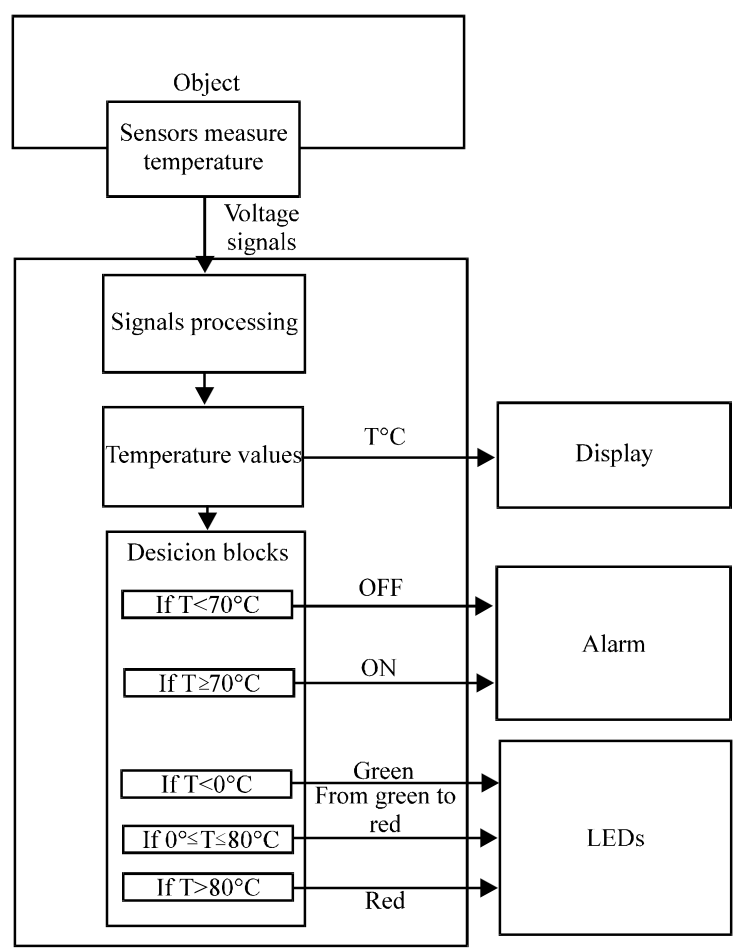

Fig. 1: General diagram of the temperature sensor response system

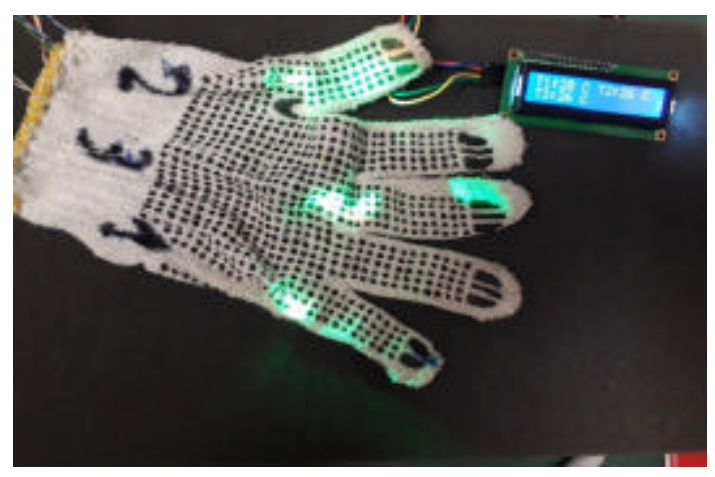

Fig. 2: Prototype of the temperature glove

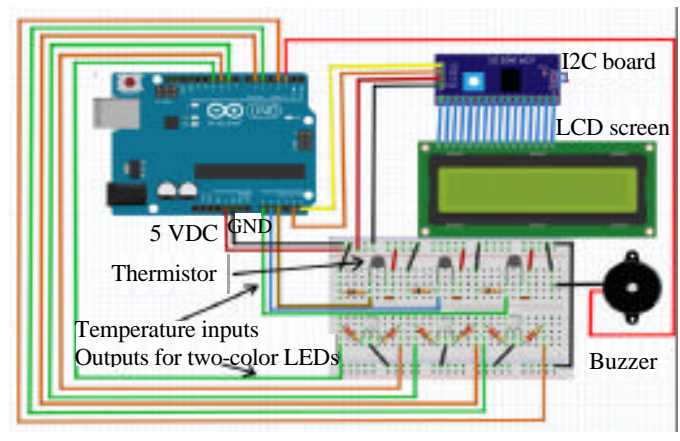

Fig. 3: Electronic circuit installed with Arduino Uno 


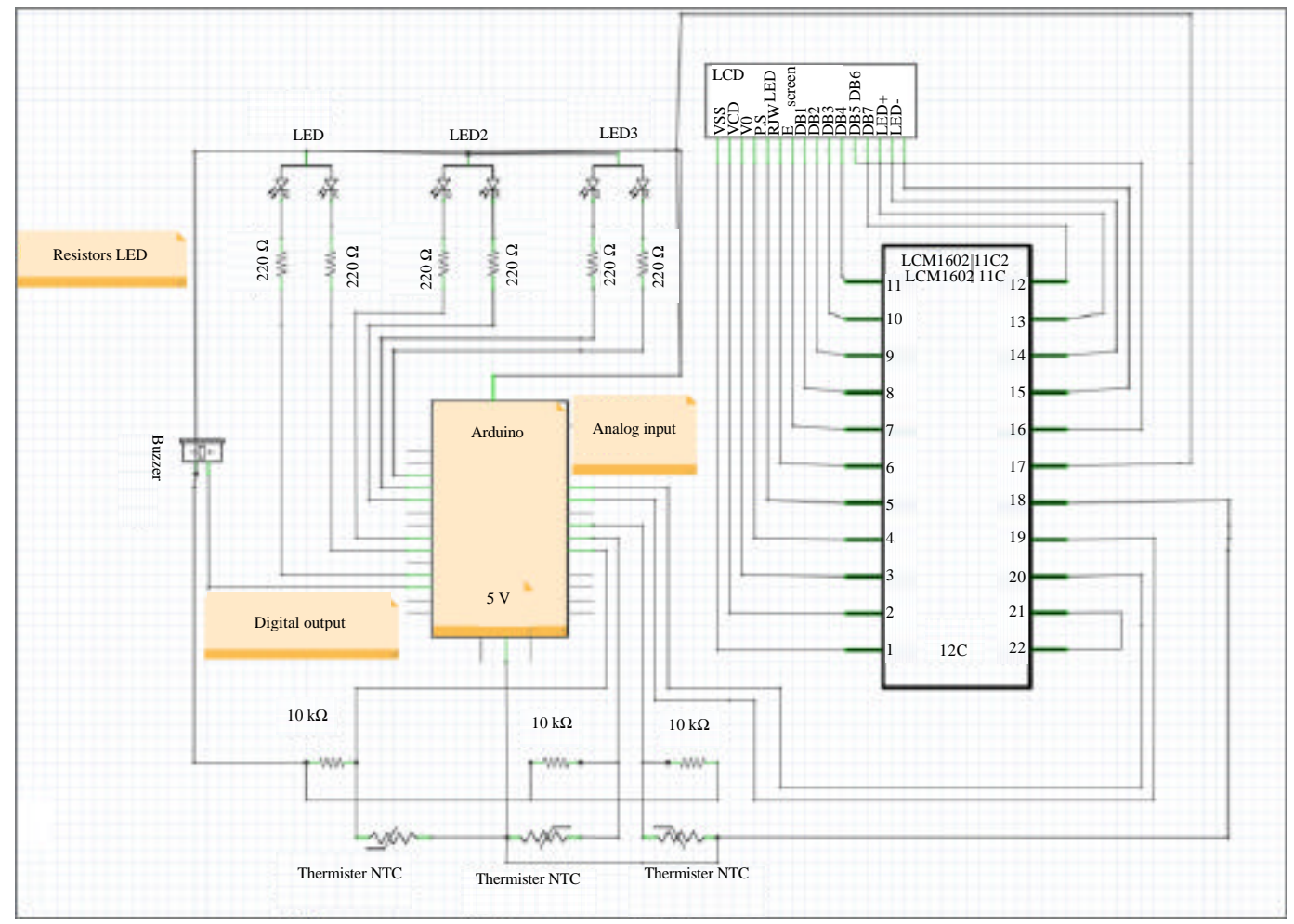

Fig. 4: Details of the electronic circuit

The $5 \mathrm{~V}$ and GND (ground) ports in the Arduino Uno board are connected to the breadboard where a first-shunt arrangement of three $10 \mathrm{k} \Omega$ thermistors and $10 \mathrm{k} \Omega$ resistance are connected to the GND with the middle pins used as voltage dividers to send the signals to analog ports A0 (S1), A1 (S2) and A2 (S3). Then, the two-color LEDs are connected to the ground of the breadboard and each pin is connected to a protection resistance of $220 \Omega$ and taken to the digital port's PWM (Kane et al., 2009; Makino et al., 11996; Sjostrom, 2001). Another output element is the buzzer which is connected to the ground and taken to a digital port (Kane et al., 2008). Finally, the LCD screen is connected to the Arduino UNO an I2C board which has the function of reducing the number of connections to only four (GND-GND, Vcc-5 V, SDA-A4 and SCL-A5).

The LEDs respond to the temperature recorded by the glove sensors. The possibilities of modifying the configurations for the user are based on two-color LEDs that change in response to temperature. The electronic circuit has been programmed to indicate unsafe (high) temperatures with a red light. The initial design is a prototype that works with temperatures ranging from $55-125^{\circ} \mathrm{C}$ with adequate sensitivity to warn users of danger. An LCD screen is connected to the Arduino board through an integrated I2C which allows us to go from 16 outputs to only 4 . With the LCD it is possible to observe the values of each Temperature sensor (T1-T3), updating each second and providing the glove with the capacity to measure the temperature of industrial equipment. The alarm of the system is a buzzer which is linked to the changes of the three thermistors at the same time. This buzzer works once the temperature sensors exceeds the limit which in our case is $70^{\circ} \mathrm{C}$. This limit can be modified to optimize the gloves for each user's needs.

The electronic circuit shown in Fig. 4 is powered by a $5 \mathrm{~V}$ DC power supply, supplying the Arduino board through a USB connection or an external battery. Other electronic elements used in the application are the $10 \mathrm{k} \Omega$ thermistors which change their resistance value as a function of temperature in an inverse way by means of a voltage divider arrangement. These voltage values are sent as a function of temperature to the Arduino board. The two-color LEDs are output elements that receive PWM signals from Arduino digital pins and through a protection resistance, we can have variable brightness depending on the temperature. An LCD screen is also incorporated through the $\mathrm{I} 2 \mathrm{C}$ coupling which is a port converter that optimizes the need for 16 pins to only 4 
pins that reach the Arduino board to obtain the temperature information in alphanumeric form. This coupling has a potentiometer that controls the brightness of the LCD screen. Finally, we have the buzzer which is a digital output of Arduino which has two states: off (temperature $<70^{\circ} \mathrm{C}$ ) and ignition (any of the three temperatures $\geq 70^{\circ} \mathrm{C}$ ). Other elements were required for the calibration and tests of the prototype including a portable temperature calibrator brand Ametek ETC400A, a hot-air blower of $1600 \mathrm{~W}$ and air volume capacity of $240 / 450 \mathrm{~L} / \mathrm{min}$ a timer and a glove holder to hold the glove during the tests.

\section{RESULTS AND DISCUSSION}

In this study, the operation of the designed glove is tested for different temperatures. Two tests were conducted considering two gloves with the same configuration one glove that sensed temperature with the three $10 \mathrm{k} \Omega$ thermistors and the other with three LM335 sensors. Each glove was subject to the tests performed with the hot-air blower and the Ametek ETC400A (Gollner et al., 2012) from $30-40^{\circ} \mathrm{C}$.

This test compared the two types of temperature sensors installed in the gloves. The first sensor is a $10 \mathrm{k} \Omega$ thermistor and the second is a precision sensor LM335 with characteristics $4.90 \times 3.91 \mathrm{~mm}$. For the test, two gloves (one with three thermistor sensors and one with three LM335 sensors) are used to observe their behavior under increasing temperature. The sensors are placed on the front and top, corresponding with each finger on the glove. For the test, the gloves are subjected to hot air with a hot-air blower for $20 \mathrm{sec}$ and then removed, so that, they can be stabilized. The sensing of the signals coming from each type of temperature sensor is made to identify its response to continuous temperature changes.

Figure 5 shows the responses of both temperature sensors during $80 \mathrm{sec}$. This test consisted of increasing the temperature linearly during the first $20 \mathrm{sec}$ and then reducing the temperature linearly to $0^{\circ} \mathrm{C}$ during the next $60 \mathrm{sec}$. Figure $5 \mathrm{a}$ shows the behavior of the glove with the thermistor sensors and Fig. 5b shows the behavior of the temperature registered in the glove with the LM335 sensors. The results show that both sensors record the temperature increase when subjected to heat with the hot-air blower.

Figure 5a shows that thermistors sense the temperature linearly, however, the sensor registered from the beginning a small environmental heat signature. Besides, the three thermistors registered, similar, temperatures with small differences among them because
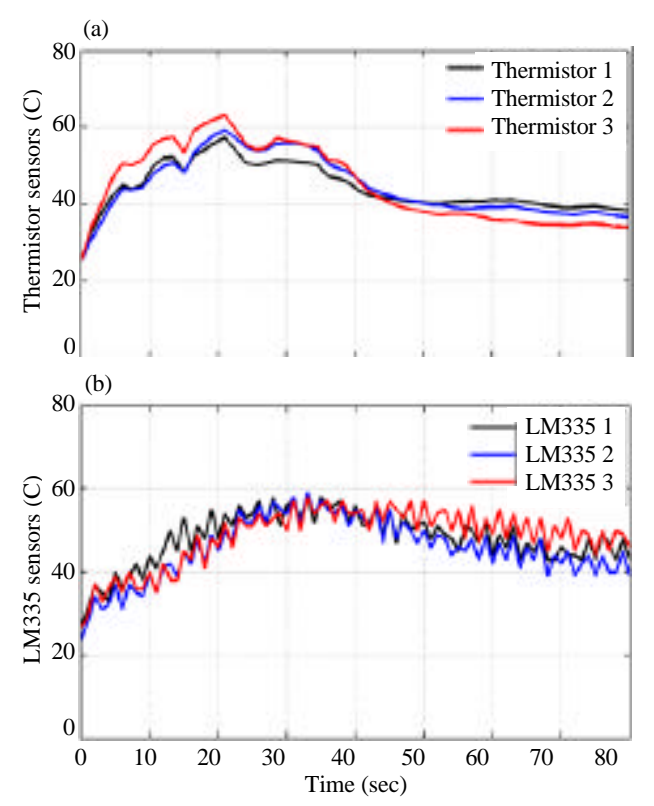

Fig. 5: Comparison of signal measured for temperature sensors subject to a hot-air blower: a) Thermistor and b) LM335

the sensors are receiving the hot air from the blower separately. Figure 5b shows that the three LM335 sensors store even more heat than the thermistor sensors and that they have a longer time delay. Additionally, these devices have a variable response over time and allow the reading to change constantly which presents a noise. However, their average value shows that the sensors measure the temperature increasing while subjected to the hot air and they register a temperature decrease when not subjected to hot air.

The following test consisted of subjecting gloves to two temperatures $\left(30\right.$ and $40^{\circ} \mathrm{C}$ ) with an Ametek type ETC-400A 12 commonly used to calibrate sensors. The gloves and sensors are subjected to temperature changes to observe their response over time and stabilization. Figure 6 shows the response of the gloves with thermistors and LM335 sensors when subjected to a temperature of $30^{\circ} \mathrm{C}$ and applied for close to $60 \mathrm{sec}$. Figures $6 \mathrm{a}$ and $\mathrm{b}$ show that the thermistors reach the temperature in less time than the LM335 sensors and the signal is more stable. The thermistors present less variation to electronic noise and electromagnetic interference than the LM335 as shown in Fig. 6.

Figure 7 shows the response of the thermistor and LM335 sensors subjected to a temperature of $40^{\circ} \mathrm{C}$ applied for $60 \mathrm{sec}$. This new test registered the responses of the sensors to a sudden change in temperature. The results show that the behavior of the sensors before the abrupt 

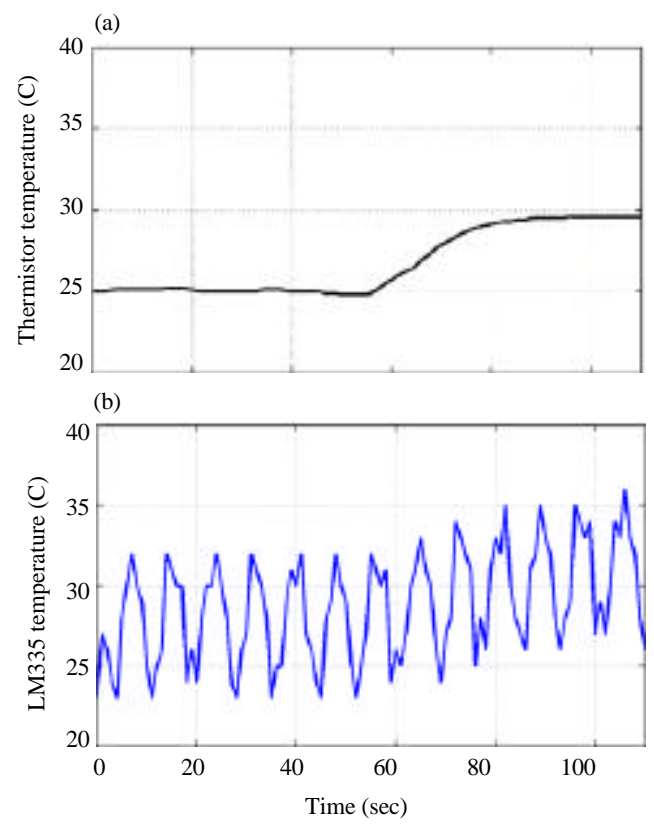

Fig. 6: Comparison of gloves subjected to a temperature change from $25-30^{\circ} \mathrm{C}$ : a) Thermistor and b) LM335
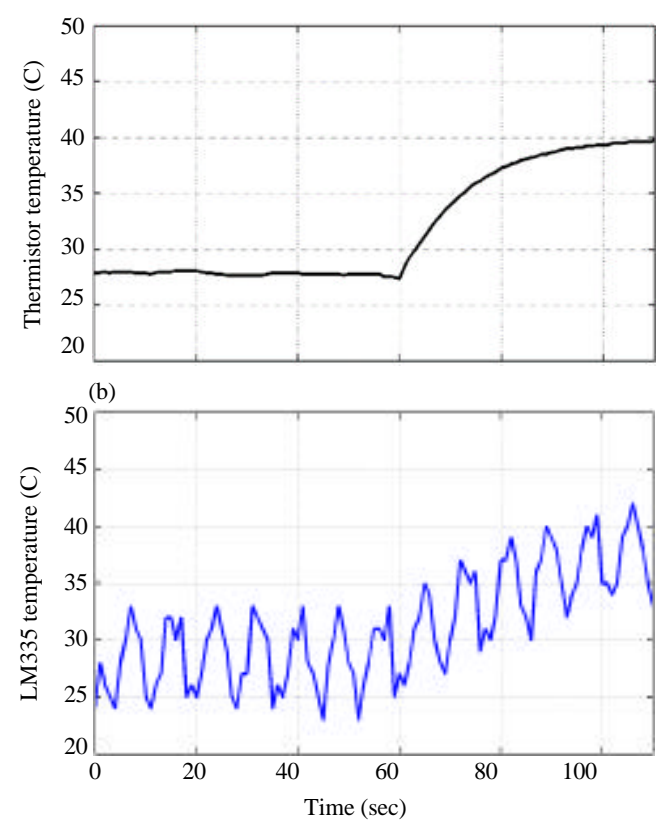

Fig. 7: Glove subjected to a temperature of $40^{\circ} \mathrm{C}$ : a) Thermistor and b) LM335

increase in temperature is the same and both reach the temperature after $40 \mathrm{sec}$. However, Fig. 7a shows that the thermistors measure the temperature better and are more stable. Therefore, the LM335 is not the appropriate sensor for the prototype presented in this research.

\section{CONCLUSION}

This study compared the performance of two temperature sensors used in high-temperature alert gloves for hand safety and injury prevention of people with visual or touch sensory disabilities. This glove alerts users when the glove comes into contact with high-temperature materials. The behaviors of the two types of temperature sensor were compared under different scenarios, concluding that these temperature sensors are very efficient in the range between 15 and $90^{\circ} \mathrm{C}$. The integration times are sufficient for the user to process the warning signals and take action. The LEDs and the audible alarm were easy to configure in order to be more adjustable to the needs of each user.

Thermistors are useful to quickly measure the temperature because they have better sensitivity to temperature changes and they offer a more accurate temperature reading. At ambient temperature, the thermistor measures very well and the LM335 fluctuates too much. The gain of the thermistor is linear and stable whereas the LM335 presents a no linear gain and unstable fluctuations. The LM335 measure includes noise which affects the accuracy. To sum up, the results showed that the thermistor is more efficient to measure the temperature under the conditions tested, occupies a smaller volume, responds more quickly and presents less error in measurement than the LM335 sensor.

An acceptable performance of the temperature sensors located in the glove was obtained during the test, monitoring the temperature and transmitting to an LCD screen with $1 \mathrm{sec}$ measurement. A satisfactory response of the sound alarm was also obtained which activated correctly at $70^{\circ} \mathrm{C}$. Finally, good performance was observed in the brightness of the two-color LEDs. Another important factor in this project is the speed of the Arduino which allows efficient temperature measurement and rapid transmission to the output pins (LEDs, LCD and buzzer). Besides, an easy configuration of the parameters established in the alarms (sound and visual) were configured to adapt to the needs of users and for future devices that make this project a more efficient tool for people with sensory disabilities.

\section{ACKNOWLEDGEMENT}

This research was supported by the Universidad Nacional de Colombia, Sede Medellín, under the projects 
HERMES-34671 and HERMES-36911. The researchers thank to the School of Physics and the Department of Electrical Energy and Automation of the Universidad Nacional de Colombia for the valuable help to conduct this research. This research was funded by the Universidad Nacional de Colombia ,Sede Medellín, under the projects HERMES-34671 and HERMES-36911.

\section{REFERENCES}

D'Atri, E., C.M. Medaglia, A. Serbanati and U.B. Ceipidor, 2007. A system to aid blind people in the mobility: A usability test and its results. Proceedings of the 2 nd International Conference on Systems, April 22-28, 2007, Martinique, France, pp: 35-35.

Elmannai, W. and K. Elleithy, 2017. Sensor-based assistive devices for visually-impaired people: Current status, challenges and future directions. Sens., 17: 1-42.

Gollner, U., T. Bieling and G. Joost, 2012. Mobile Lorm Glove: Introducing a communication device for deaf-blind people. Proceedings of the 6th International Conference on Tangible, Embedded and Embodied Interaction, February 19-22, 2012, ACM, Kingston, Canada, ISBN:978-1-4503-1174-8, pp: 127-130.
Kane, S.K., C. Jayant, J.O. Wobbrock and R.E. Ladner, 2009. Freedom to roam: A study of mobile device adoption and accessibility for people with visual and motor disabilities. Proceedings of the 11th International ACM SIGACCESS Conference on Computers and Accessibility, October 25-28, 2009, ACM, Pittsburgh, Pennsylvania, ISBN: 978-1-60558-558-1, pp: 115-122.

Kane, S.K., J.P. Bigham and J.O. Wobbrock, 2008. Slide rule: Making mobile touch screens accessible to blind people using multi-touch interaction techniques. Proceedings of the 10th International ACM SIGACCESS Conference on Computers and Accessibility (Assets '08), October 13-15, 2008, ACM, Halifax Regional Municipality, Canada, ISBN:978-1-59593-976-0, pp: 73-80.

Makino, H., I. Ishii and M. Nakashizuka, 1996. Development of navigation system for the blind using GPS and mobile phone combination. Proceedings of the 18th Annual International Conference on the IEEE Engineering in Medicine and Biology Society, October 31-November 3, 1996, IEEE, Amsterdam, Netherlands, pp: 506-507.

Sjostrom, C., 2001. Designing haptic computer interfaces for blind people. Proceedings of the 6th International Symposium on Signal Processing and its Applications (Cat.No.01EX467), August 13-16, 2001, IEEE, Kuala Lumpur, Malaysia, pp: 68-71. 